\title{
Defined Criterion Group Option Relationship Pause Quantity
}

National Cancer Institute

\section{Source}

National Cancer Institute. Defined Criterion Group Option Relationship Pause Quantity.

NCI Thesaurus. Code C93766.

A quantity of time that should elapse between when an activity is ready for execution and the actual beginning of the execution. 